УДК 621.314.1:621.382.2

\title{
БИФУРКАЦИОННЫЕ ЯВЛЕНИЯ В ПРЕОБРАЗОВАТЕЛЕ НАПРЯЖЕНИЯ С ЧАСТОТНО-ИМПУЛЬСНЫМ УПРАВЛЕНИЕМ ДЛЯ ВЕТРОГЕНЕРАТОРНОЙ УСТАНОВКИ
}

\author{
Михальченко Сергей Геннадьевич 1 , \\ msg@ie.tusur.ru
}

Михальченко Геннадий Яковлевич 1

\section{Семенов Сергей Михайлович²,} ssm@tpu.ru

\author{
Мещеряков Павел Сергеевич ${ }^{\text {, }}$ \\ mps@ie.tusur.ru
}

\section{Воронина Наталья Алексеевна² voronina@tpu.ru}

\author{
Зюзев Анатолий Михайлович ${ }^{3}$, \\ a.m.zyuzev@urfu.ru \\ 1 Томский университет систем управления и радиоэлектроники, \\ Россия, 640050, г. Томск, пр. Ленина, 40. \\ 2 Национальный исследовательский Томский политехнический университет, \\ Россия, 640050, г. Томск, пр. Ленина, 30. \\ 3 Уральский федеральный университет, \\ Россия, 620002, г. Екатеринбург, ул. Мира, 19.
}

Статья посвящена изучению влияния бифуркационных явлений, имеющих место во всех типах импульсно-модуляционных систем, которые в частности присутствуют в ветрогенераторных установках. Для исследования динамики преобразователя напряжения с частотно-импульсной модуляцией строится математическая модель и бифуркационные диаграммы, которые иллюстрируют область безопасной работы ветрогенераторных установок, проводится ряд численных экспериментов. Выявлен новьй вид бифуркационного явления, названый в данной статье «пакетной» бифуркацией.

Актуальность работы обуславливается необходимостью улучшения энергоэфффективности силовых преобразовательных устройств для потребностей нетрадиционной энергетики, в частности - для ветрогенераторной установки. Одна из сложностей преобразования данного вида энергии заключается в невысоком уровне эфффективности процесса. Ввиду переменной величины частоты вращения лопастей ветрогенераторной установки в определенном временном интервале параметры получаемой энергии также варьируются, следовательно, настройки её вторичных преобразователей должны соответствовать временным интервалам формирования сигнала генерации. Повышение качества ффункционирования альтернативных источников питания возможно за счет использования многочастотных режимов стабилизации устройства преобразования. В статье рассматривается модель импульсно-модуляционного понижающего преобразователя напряжения на основе частотно-импульсной модуляции. Изучены и предложены методики изменения ширины рабочей зоны одночастотного режима стабилизации.

Цель: анализ режимов вторичного преобразователя напряжения ветрогенераторной установки и выработка подходов к исследованию бифуркационных явлений, имеющих место в преобразовательных структурах с частотно-импульсной модуляцией путем построения математической модели и численно-аналитического моделирования данного понижающего устройства для улучшения показателей надежности.

Методы: проработка литературных источников по типовым схемам передачи энергии в ветрогенераторную установку с промежуточным звеном постоянного тока (DC/DC преобразователей); математическое моделирование и проведение численных экспериментов по изменению эксплуатационных данных понижающего преобразователя напряжения $c$ частотноимпульсной модуляцией для выявления протекающих в нем бифуркационных процессов; построение биффуркационных диаграмм при различных параметрах преобразователя с целью повышения надежности работы.

Результаты. Получены математические модели и численно-аналитическая методика построения с идентификацией динамических режимов функционирования понижающего преобразователя напряжения с частотно-импульсной модуляцией, выступающего в роли звена постоянного тока ветрогенераторной установки; построены бифуркационные диаграммы для стабилизации напряжения при различных параметрах системы управления; показано одновременное наличие одночастотных и пакетных режимов работы; идентифицирована бифруркация деления периода; изучено влияние параметров устройства на режимы работы, а также предложена методика изменения ширины рабочей зоны одночастотного режима стабилизации.

Ключевые слова:

Ветрогенерация, понижающий преобразователь напряжения, частотно-импульсная модуляция, коммутационная функция, бифуркационная диаграмма, одночастотный режим стабилизации. 


\section{Введение}

Глобальный совет по ветроэнергетике (Global Wind Energy Council - GWEC) опубликовал итоговый отчет о ежегодном обновлении рынка ветроэнергетики (Global Wind Report: Annual Market Update). В нем говорится, что в мировом масштабе в 2016 г. установлено более 54 ГВт источников чистой возобновляемой энергии ветра в более чем 90 странах. Из них девять государств (рис. 1) увеличили соответствующего вида мощности за 10000 МВт, а 29 прошли отметку 1000 МВт [1].

Генеральный секретарь GWEC Стив Сойер заявил: «Власть ветра теперь успешно конкурирует с сильно субсидируемыми операторами по всему миру, создавая новые отрасли промышленности, сотни тысяч рабочих мест и направляя человечество к будущему чистой энергии. В энергетике сейчас доминирует целый ряд широко распространенных возобновляемых источников энергии. Наша цель - получить систему электроэнергии с нулевой эмиссией до 2050 года, если мы хотим достичь наших целей в области изменения климата и развития» [1].

На рис. 2 приведена суммарная емкость рынка ветроэнергетики, возросшая к концу 2017 г. на 12,6 \% и достигнувшая в общей сложности 486,8 ГВт.

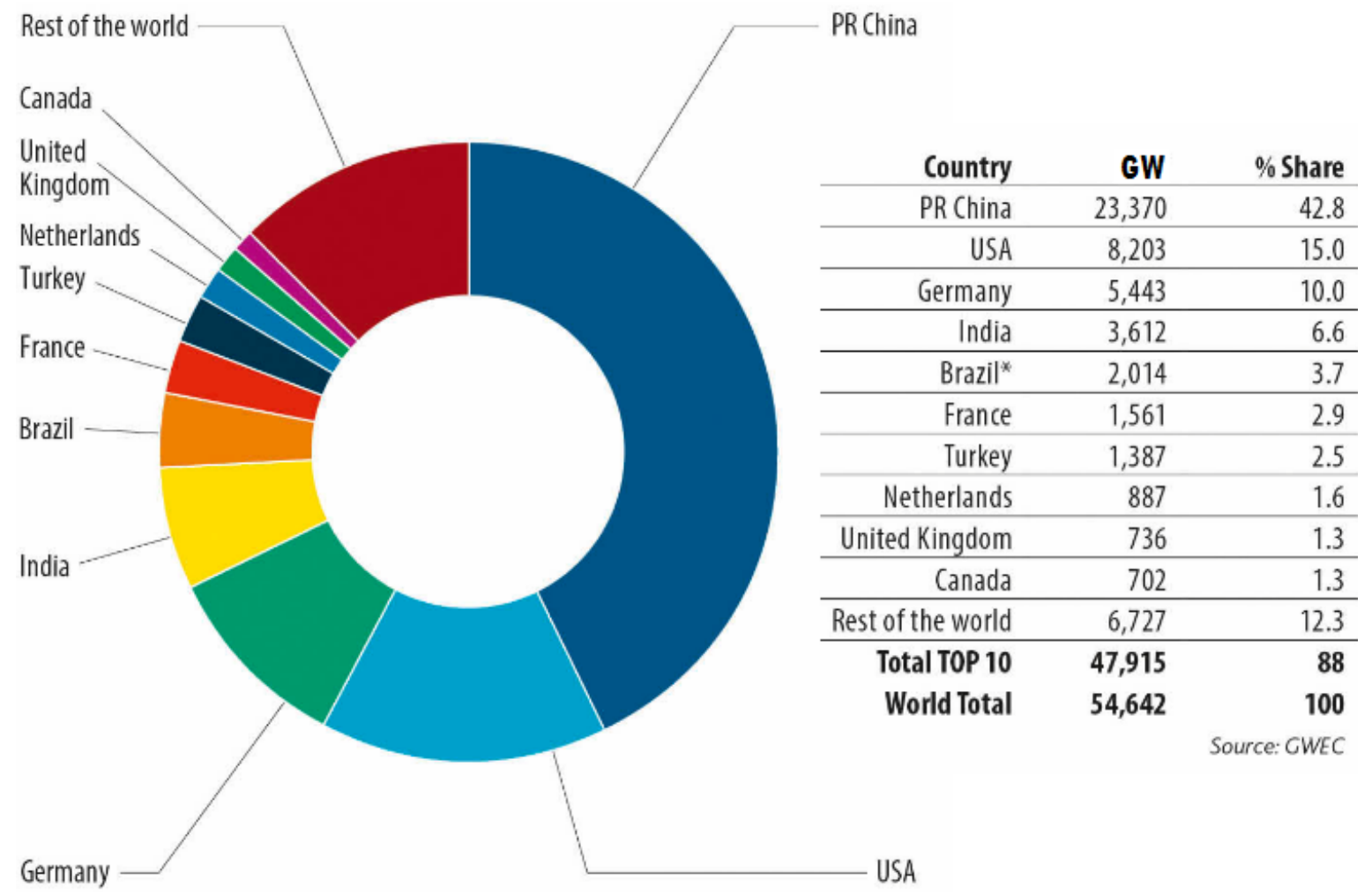

Puc. 1. Ветроэнергетика: десятка лидеров установки новых мощңностей в 2016 2. [1]

Fig. 1. Wind power engineering: ten leaders in new capacity installation in 2016 [1]

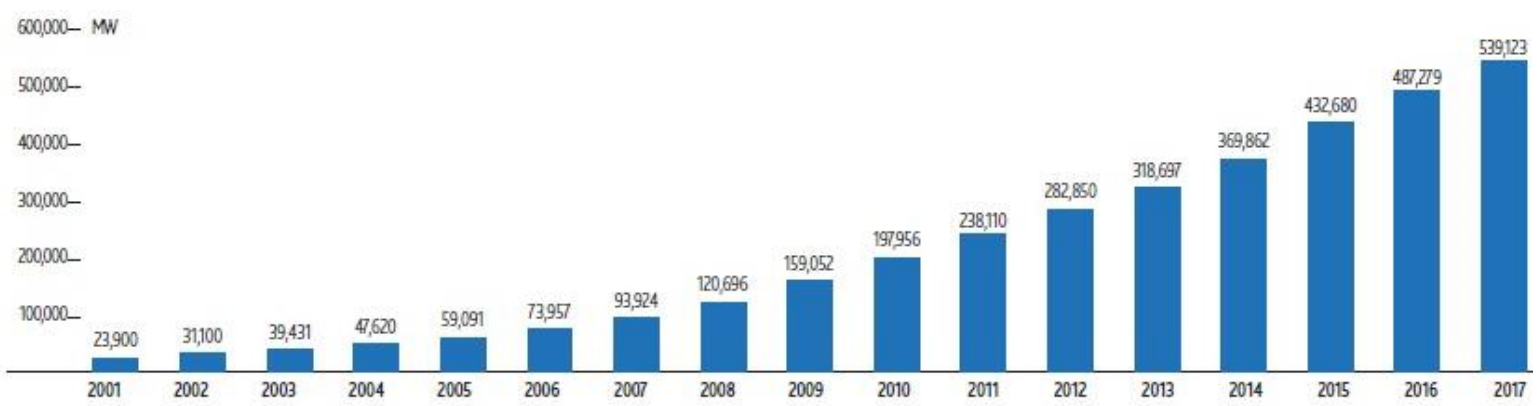

Pис. 2. Ветроэнергетика: совокупная установленная мощңность 2001-2017 г2. [1]

Fig. 2. Wind power engineering: the total installed capacity in 2001-2017 years [1]

Каково же положение российской ветроэнергетики в мире? Россия включилась в число стран, поддерживающих технологии возобновляемых источников энергии (ВИЭ) в 2013 г., когда вышло постановление Правительства РФ от 28 мая 2013 № 449 «О механизме стимулирования использования возобновляемых источников энергии на оптовом рынке электрической энергии и мощности» [2]. Действие данного постановления было продлено распоряжением Правительства РФ от 05 мая 2016 № 850-р «О продлении программы поддержки ВИЭ до 2024 года» [3]. 
Этими законодательными актами сформулированы перспективы развития оптового рынка ветрогенерации в России: до 2024 г. будет построено 3600 МВт ветропарков, причем это довольно скромная величина Достигнув её, страна даже вряд ли попадет в десятку мировых лидеров развития ВИЭ. Значит, нет сомнений в необходимости повышения эффективности работы в данном направлении [4].

В ветроэнергетике за последние 10 лет стоимость выработки киловатт-часа упала в три раза за счет увеличения объема выпуска ветрогенераторов и применения более совершенных моделей.

Действующие в России меры поддержки ВИЭ создают возможности для развития этого направления, в том числе для местного производства соответствующего оборудования. В то же время точечные меры не могут в полном объёме компенсировать недостатки среды в целом. Высокая стоимость капитала, дефицит финансовых ресурсов и начинающаяся рецессия 2020 г. затрудняют промышленное развитие России, в том числе и ветроэнергетическое машиностроение.

На данном этапе перспективным является рост возобновляемых источников энергии на местном уровне, в коллективных и индивидуальных хозяйствах, в том числе и автономных вариантах. Последний пункт служит хорошим источником для развития ветроэнергетики в регионах. В ситуации, когда цен- трализованной энергией обеспечено только 40 \% территории России, ВИЭ можно считать серьезной альтернативой «северному завозу» - транспортировкам энергетических ресурсов в удаленные районы. Для автономных хозяйств, удаленных от централизованных ВИЭ, это тройная выгода: социальная, экономическая и экологическая [5].

\section{Обоснование выбора системы}

управления ветроустановкой

Достаточно большое количество научных статей по направлению «ветрогенерация» посвящены анализу вариантов конструктивных решений ветроустановок, параметров воздушных потоков [6, 7], и это понятно - существенные потери содержит их механическая составляющая. Однако стоит обратить внимание и на электрическую преобразовательную часть устроиств [8].

Основные отличия схем элетрических соединений состоят в конструкции и типе генераторов [9], а также в наличии регулирующих аппаратов. Для систем ветрогенерации средней и малой мощности с повышенными показателями качества и техническими параметрами, как правило, применяются структуры с узлами выпрямления и инвентирования (рис. 3) или частотного регулирования сигнала. Данная статья посвящена использованию частотно-импульсной модуляции (ЧИМ) в звене постоянного тока (DCLDC).

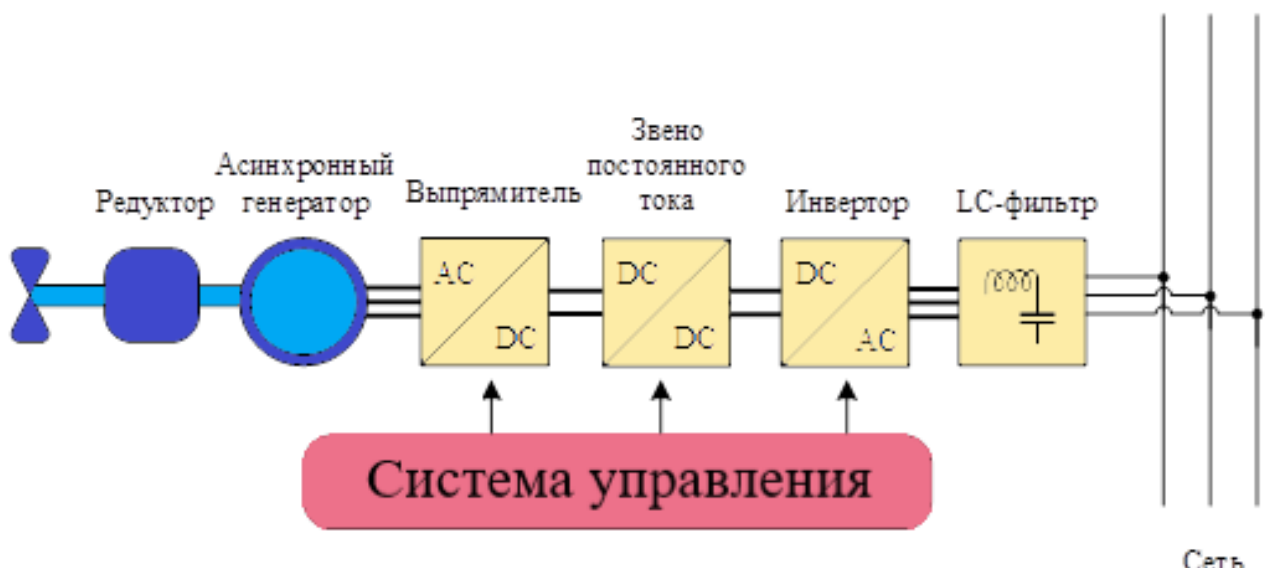

Pис. 3. Типовая структура ветрогенераторной установки [9]

Fig. 3. Typical structure of a wind generator set [9]

Применение именно ЧИМ в непосредственном стабилизаторе напряжения обусловлено наличием высших гармоник в выпрямленном сигнале, поступившем от электрогенератора [10]. Перед промежуточным высокочастотным преобразователем напряжения (ПН) ставится кроме этого еще ряд задач: отбор максимальной мощности (Maximum Power Point Tracking, MPPT) $[11,12]$, электромагнитной совместимости (Electromagnetic Compatibility, EMC) и caмая главная - стабилизации уровня сигнала [13].

Сложности, возникающие на этом этапе, в основном связаны с нелинейностью и аномальностью динамики импульсно-модуляционных преобразователей. Авторам не встречались устройства такого типа, для которых в пространстве рабочих параметров отсутствовали бы аномальные режимы работы. Не стал исключением и преобразователь напряжения с ЧИМ, выполняющий роль звена постоянного тока ветрогенераторной установки $[14,15]$.

Исходя из сказанного, делается попытка выявить возможные недокументированные аномальности в работе ПН с ЧИМ посредством бифуркационного анализа. Для этого применяется параметрический синтез карты динамических режимов ПН с ЧИМ данной структуры с целью получить характеристики запаса устойчивости преобразователя и его динамические свойства с точки зрения внешних воздействий $[16,17]$. 


\section{Анализ математической модели преобразователя}

За основу исследования была выбрана математическая модель понижающего DC/DC-преобразователя напряжения, анализ нелинейной динамики с широтно-импульсным управлением которым приведен в $[16,17]$. Он относится к системам с частотноимпульсной. На рис. 4 изображена схема замещения понижающего преобразователя с ЧИМ.

При построении модели приняты допущения аналогичные приведенным в источниках [14-17].

Приведенная схема описывается математической моделью в виде задачи Коши для обыкновенных дифференциальных уравнений.

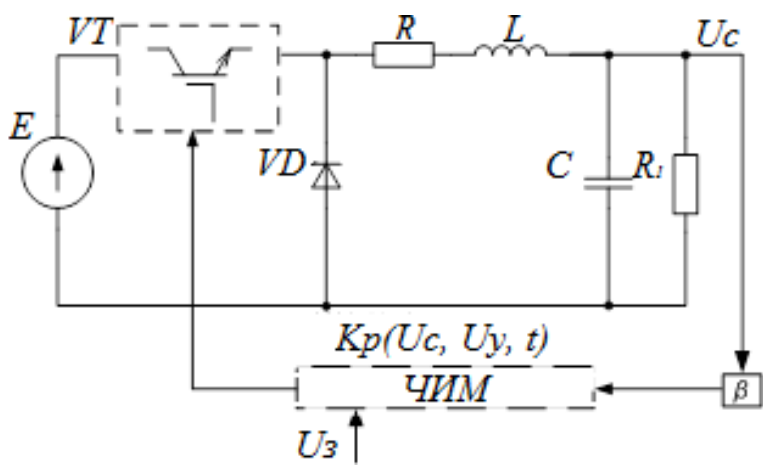

Рис. 4. Схема замещения понижающего преобразователя с ЧИМ

Fig. 4. Electrical diagram of buck converter with a pulsefrequency modulation (PFM)

В состав рассматриваемой схемы входят: $E$ входное напряжение преобразователя; VT - полупроводниковый ключ; $V D$ - диод; $R, L, C$ - параметры непрерывной части, где $R$ - характеризует омические потери дросселя фильтра, а также сопротивление ключа в открытом состоянии, $R_{\mathrm{H}}-$ сопротивление нагрузки; $\beta$ - коэффициент передачи датчика обратной связи по напряжению $U_{C} ; U_{3}$ - задающее напряжение; ЧИМ - частотно импульсный модулятор.

Задача Коши для поиска периодического решения $\mathrm{X}(t)=\mathrm{X}(t+T)$ может быть поставлена в матричной форме:

$$
\left.\begin{array}{c}
\frac{d \mathrm{X}}{d t}=\mathrm{A}(K F(\Theta), \mathrm{X}) \cdot \mathrm{X}+\mathrm{B}(K F(\Theta), \mathrm{X}), \\
\mathrm{A}=\left[\begin{array}{cc}
-\frac{R}{L} & -\frac{1}{L} \\
\frac{1}{C} & -\frac{1}{C \cdot R_{\mathrm{H}}}
\end{array}\right], \mathrm{B}=\left[\begin{array}{c}
\frac{E}{L} \\
0
\end{array}\right], \\
\mathrm{X}=\left[\begin{array}{c}
i_{L} \\
U_{C}
\end{array}\right], \mathrm{X}\left(t_{0}\right)=\mathrm{X}_{0}, \mathrm{X}(t)=\mathrm{X}(t+T)
\end{array}\right\}
$$

где X - вектор неизвестных; А - квадратная матрица параметров линейной части, характеризующая схему замещения; В - вектор внешних воздействий [17].

Функция $K F(\Theta)$, описывающая управляющую импульсную последовательность, является кусочнолинейной, таким образом, имеется возможность представить результат с использованием уравнения (1) на участках линейности $K F$.

\section{Исследование параметров ЧИМ}

Коммутационная функция $K F(\Theta)$ формируется блоком ЧИМ. Опишем логику его работы с изображением в виде структуры, приведенной на рис. 5.

Сигнал датчика обратной связи $\beta \cdot U_{\mathrm{c}}(t)$ поступает на входное устройство, там же задается коэффициент усиления регулятора $\alpha$. Этот блок является источником тока, управляемым напряжением (ИТУН), при этом регулирующим воздействием служит выражение ошибки $\alpha\left(U_{3}-\beta \cdot U_{\mathrm{c}}(t)\right)$.

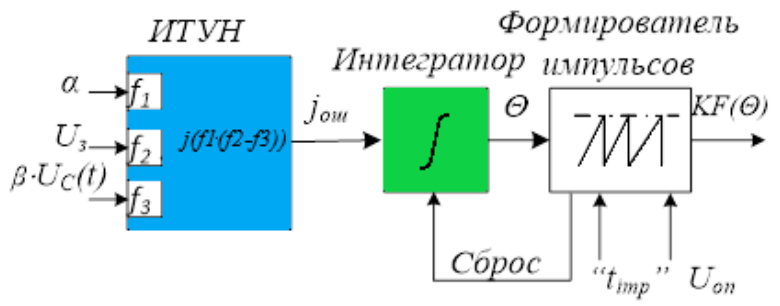

Puc. 5. Функииональная схема ЧИМ

Fig. 5. Chain diagram of the PFM

Желаемое выходное воздействие силового преобразователя задается напряжением $U_{3}$. ИТУН генерирует токовый импульс $j_{\text {ош, }}$ соответствующий уровню ошибки по напряжению, значения $j_{\text {ош }}$ ограничены минимальными и максимальными величинами. Далее $j_{\text {ош }}$ подается на блок интегратора, сигнал интегрирующего звена $\Theta(t)$ поступает на формирователь импульсов. Данный элемент представляет собой компаратор напряжения, срабатывающий по достижению

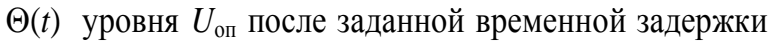
$t_{\text {imp. }}$ Формируемый при этом импульс заданной длительности поступает на драйвер силового транзистора и одновременно является сигналом для сброса уровня интегратора.

На рис. 6 представлены графики, поясняющие алгоритм работы ЧИМ. Из него видно, что чем больше ток $j_{\text {ош }}$, тем выше частота $K F(\Theta)$. Длительность импульса есть постоянная величина, меняется только частота сигнала управления. Временные интервалы задержки срабатывания формирователя импульса и его длина равны. В момент $(k-1) \tau$ включается интегратор, и за некоторое время $t_{\text {uр }}$ сигнал $\Theta(t)$ достигает опорного напряжения $U_{\text {оп }}$ (момент времени $z_{n}$ ), в период временной задержки $t_{\text {imp }}$ значение напряжения $\Theta(t)$ продолжает нарастать до уровня $U_{\mathrm{C} \Theta}$ (или до $U_{C \max }$ при $\left.j_{\max }\right)$, после $t_{\text {imp }}$ происходит сброс напряжения интегратора. В момент $k \tau$ данный блок снова включается и процессы повторяются.

В рассматриваемой математической модели сигнал $\Theta(t)$ моментально сбрасывается во время формирования импульса, а в реальных системах (если будет использован емкостной накопитель) сброс энергии производится в течение конечного периода времени,

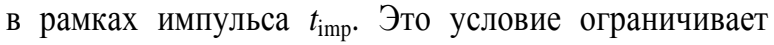
разработчика с одной стороны по параметрам конденсатора, применяемого в интеграторе, с другой - по длительности интервала $t_{\mathrm{imp}}$. 


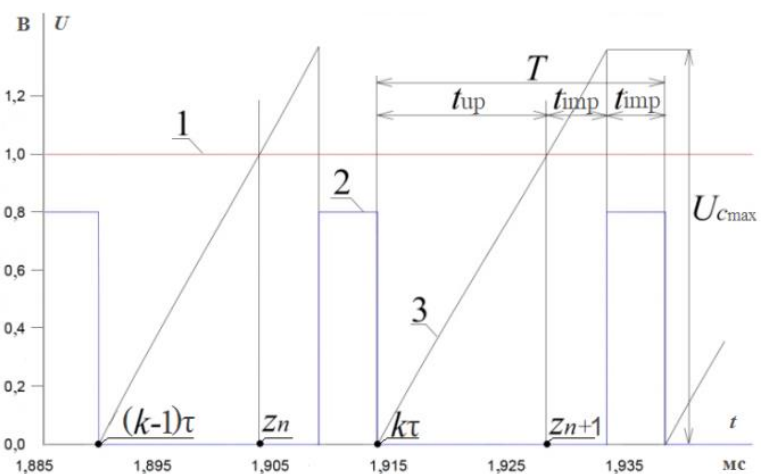

Pис. 6. Диаграммы формирования импульсной последовательности: 1 - опорное напряжение $U_{\text {оп; }} 2$ импульсная последовательность KF; 3 - сигнал интегратора $\Theta$

Fig. 6. Diagrams of pulse sequence formation: 1 - reference voltage $U_{o n} ; 2$ - pulse sequence $K F ; 3$ - integrator signal $\Theta$

На основе функциональной схемы (рис. 5) разработана имитационная модель ЧИМ $[12,14,18]$ с реализацией в имитационном комплексе LTSpice IV (Linear Technology, Co).

Далее приводится математическое выражение, описывающее работу модулятора. Токовый сигнал ошибки, генерируемый ИТУН (рис. 5), математически записывается следующим выражением:

$$
j_{\text {ош }}(t)=\alpha \cdot\left(U_{3}-\beta \cdot X 2(t)\right), j_{\min }<j(t)<j_{\max },
$$

где $j_{\min }, j_{\max }-$ минимальный и максимальный токи ИТУН; $\alpha$ - коэффициент усиления сигнала ошибки; $\left(U_{3}-\beta \cdot X 2(t)\right)$ - значение сигнала ошибки; $U_{3}-$ задающее напряжение; $X 2(t)$ - моментальная величина напряжения на выходе преобразователя - воздействия, получаемого по обратной связи.

Интегрирование токового импульса осуществляется посредством заряда конденсатора:

$$
\Theta(t)=\frac{1}{C_{\text {sum }}} \int_{0}^{\mathrm{t}} j_{\text {ош }}(t) \cdot d t,
$$

где $C_{\text {sum }}-$ значение емкости интегрирующего конденсатора $\mathrm{C} 1$

Компаратор формирует запаздывающую коммутационную функцию и может находиться в одном из двух дискретных значений: 1 - для открытого состояния транзистора VT и 0 - для запертого. Условия данных состояний:

$$
K F(t)=\left\{\begin{array}{l}
1, \Theta(t)>U_{\text {оп }} \wedge\left(z_{n}+t_{\text {imp }}\right) \leq t \leq\left(z_{\mathrm{n}}+2 t_{\text {imp }}\right) \\
0, \Theta(t)>U_{\text {оп }} \wedge\left(t<z_{n}+t_{\text {imp }}\right) \vee \Theta(t)<U_{\text {оп }}
\end{array} .\right.
$$

Зададимся максимальной частотой управляющего сигнала согласно теореме Котельникова. Асимптотическая максимальная частота определяется параметром $t_{\text {imp }}$ и равна:

$$
f_{\max a}=\frac{1}{2 t_{\mathrm{imp}}} .
$$

Но из-за того, что система управления имеет конечную скорость нарастания сигнала интегратора, она будет работать на более низких частотах. Выведем выражения для фактической максимальной частоты функционировать ЧИМ. Чем больше система отклоняется от необходимого параметра, тем больший ток генерируется ИТУН, соответственно тем выше частота управления. Примем, что ток этого источника равен максимальному возможному $j_{\max }-$ peжим насыщения ИТУН.

Исходя из логики действия ЧИМ (его модель изложена выше) в управляющей импульсной последовательности существует два временных интервала, которые не изменяются, а именно время паузы и им-

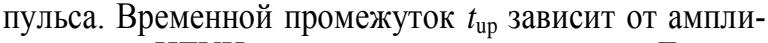
туды тока ИТУН и от емкости конденсатора. Так как ток источника принимает постоянное значение, заряд конденсатора происходит на линейном участке траектории. Период управляющего сигнала (рис. 6) скла-

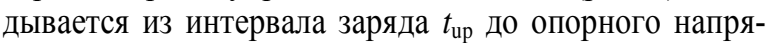
жения и из двух интервалов импульса $t_{\text {imp }}$ :

$$
T=t_{\text {up }}+2 \cdot t_{\text {imp }} \text {. }
$$

Из уравнения (4) вычислим $t_{\text {up }}$ и $t_{\text {imp }}$ :

$$
t_{\text {imp }}=\frac{C_{\text {sum }} \cdot\left(U_{C \max }-U_{\text {oI }}\right)}{j_{\text {max }}}, t_{\text {up }}=\frac{C_{\text {sum }} \cdot\left(U_{\text {оп }}-0\right)}{j_{\text {max }}} \text {. }
$$

Выражения (7) подставим в соотношение (6), тогда:

$$
T=\frac{C_{\text {sum }}}{j_{\max }} \cdot\left(2 \cdot U_{\text {Cmax }}-U_{\text {оп }}\right) .
$$

Зависимость (8) служит для расчета минимального периода сигнала управления. Из формулы (7) выразим $C_{\text {sum }}$, и, если максимальное напряжение интегри-

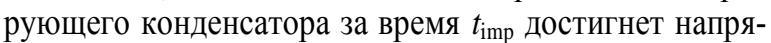
жения в $N$ раз больше опорного: $U_{C \max }=N U_{\text {оп }}$, тогда

$$
C_{\text {sum }}(N)=\frac{t_{\text {imp }} \cdot j_{\text {max }}}{U_{\text {оп }}(N-1)} .
$$

Подставим уравнение (9) в зависимость (8) и получим:

$$
T(N)=t_{\text {imp }} \cdot \frac{(2 N-1)}{(N-1)},
$$

или для частоты:

$$
f_{\max }(N)=\frac{1}{t_{\text {imp }}} \cdot \frac{(N-1)}{(2 N-1)} .
$$

Для конкретизации вычислений предположим $N=10, j_{\min }=0 \mathrm{~A}, j_{\max }=0,5 \mathrm{~A}, t_{\mathrm{imp}}=5$ мкс, тогда:

$$
\begin{aligned}
C_{\text {sum }} & =\frac{t_{\text {imp }} \cdot j_{\max }}{\left(9 \cdot U_{\text {оп }}\right)}=\frac{5 \text { мкс } \cdot 0,5 \mathrm{~A}}{9 \cdot 1 \mathrm{~B}}=277,8 \text { нФ, } \\
f_{\max } & =\frac{1}{t_{\text {imp }}} \cdot \frac{(N-1)}{(2 N-1)}=\frac{1}{5 \text { мкс }} \cdot \frac{9}{19}=94,7 \text { кГц. }
\end{aligned}
$$

Примем $C_{\text {sum }}=270$ нФ, расчетная максимальная частота $f_{\max }$ при этом равна 94,7 кГц.

Решение системы уравнений (2)-(5), (11) производится по методике построения стробоскопического отображения $[16,17]$ при следующем наборе фиксированных параметров: 


$$
P=\left\{\begin{array}{l}
E=1040 \mathrm{~B} ; R_{\mathrm{H}}=100 \text { Ом; } \\
R=10,6 \text { Ом } L=0,1 \text { Гн; } C=1 \mathrm{мк} \Phi ; \\
\beta=0,01 ; U_{3}=2 \mathrm{~B} ; \\
U_{\text {ог }}=1 \mathrm{~B} ; \alpha=2 ; j_{\max }=0,5 \mathrm{~A} ; j_{\min }=0 \mathrm{~A} ; \\
C_{\text {sum }}=270 \mathrm{H} \Phi ; t_{\text {imp }}=5 \mathrm{мкс.}
\end{array}\right\}
$$

Параметры силовой части схемы подобраны таким образом, чтобы имелась возможность сравнить динамику исследуемого преобразователя с устройством, рассмотренным в [17], не прибегая к относительным единицам.

\section{Поиск стационарных периодических режимов}

Запишем общее решение исходной задачи (1) по аналогии с исследованиями в $[16,17]$ на участках линейности, согласно условию (4), определяемых функцией $K F$ :

$$
\mathrm{X}(t)=\mathbf{e}^{\mathrm{A}\left(t-t_{0}\right)}\left(\mathrm{X}_{0}+\mathrm{A}^{-1} \mathrm{~B} \cdot K F(\Theta)\right)-\mathrm{A}^{-1} \mathrm{~B} \cdot K F(\Theta) .(13)
$$

Это решение зависит от начальных условий интегрирования $X_{0}$ и $t_{0}$ - параметры тока и напряжения $X_{0}$ на левой границе $t_{0}$ интервала линейности.

1. С учетом формулы (13), на периоде времени $(k-1) \tau \leq t \leq z_{n}$ нарастания сигнала интегратора коммутационная функция принимает значение нуля, т. е. $B=[0,0]^{T}$ и тогда:

$$
\mathrm{X}(t)=e^{\mathrm{Al} \cdot(t-(k-1) t)} \cdot\left(\mathrm{X}_{k-1}+\mathrm{A} 1^{-1} \cdot \mathrm{B} 2\right)-\mathrm{A} 1^{-1} \cdot \mathrm{B} 2 .
$$

2. На интервале паузы $z_{n} \leq t \leq z_{n}+t_{\text {imp }}$ коммутационная функция также равна нулю, т. е. $B=[0,0]^{T}$, решение на этом участке соответственно примет вид:

$$
\mathrm{X}(t)=e^{\mathrm{Al} \cdot\left(t_{\mathrm{imp}}\right)} \cdot\left(\mathrm{X}_{\mathrm{z}_{n}}+\mathrm{A} 1^{-1} \cdot \mathrm{B} 2\right)-\mathrm{A}^{-1} \cdot \mathrm{B} 2 .
$$

3. На отрезке времени $z_{n}+t_{\text {imp }} \leq t \leq z_{n}+2 t_{\text {imp }}$ коммутационная функция равна единице, т. е. $B=[E / L, 0]^{T}$, отсюда:

$$
\mathrm{X}(t)=e^{\mathrm{Al} \cdot\left(t_{\mathrm{imp}}\right)} \cdot\left(\mathrm{X}_{\mathrm{z}_{n}+\mathrm{t}_{\mathrm{imp}}}+\mathrm{A} 1^{-1} \cdot \mathrm{B} 1\right)-\mathrm{A} 1^{-1} \cdot \mathrm{B} 1 .(16)
$$

По выражениям (14)-(16) с учетом формул (3) и (4) построена математическая модель решения задачи (1) в среде Matlab. Для верификации моделирования в среде LTSpice IV, по аналогии с [16], разработана имитационная модель преобразователя.

\section{Имитационное моделирование}

Модель выполнена в среде LTSpice, представлена на рис. 7. Коммутационно-разрывная функция обратной связи .func Foc() задана в соответствии с соотношениями (2)-(4), полученные результаты учтены на рис. 8 .

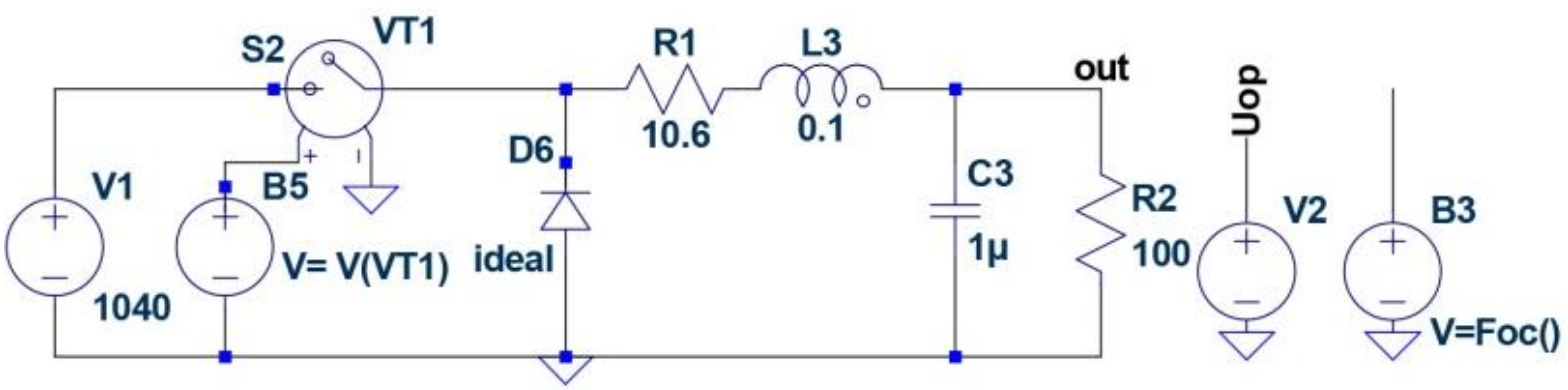

\section{.model VT1 SW(Ron $=0.0001 \mathrm{~m}$ Roff $=10000 \mathrm{Meg} \mathrm{Vt}=0.001 \mathrm{Vh}=0$ ) .model ideal $D(R o n=0$ Roff $=1000 \mathrm{Meg}$ Vfwd $=0 \mathrm{mfg}=$ ideal type=ideal .tran $020 \mathrm{~m} 0.1 \mathrm{~m} 5 \mathrm{n}$}

Pис. 7. Имитационная модель в среде LTSpice: V2 - источник задающего напряжсения; .func Fос() - функиия обратной связи; $V(U z)$ - задающее напряжение; $V($ out $)$ - выходное напряжение силовой части

Fig. 7. Simulation model in the LTSpice: V2 - the source of the set voltage; func Foc()-feedback function; V(Uz) - the set voltage; V(out) - the output voltage of the power part

Результаты имитационного и математического моделирования для двух вариантов приводятся ниже. Первый - для значения интегрирующего конденсатоpa $N=10$ и параметров блока (12). Полученная максимальная частота равна 94,64 кГц, а максимальная амплитуда заряда конденсатора - 10,1 В, что близко к расчетным величинам для опорного напряжения $U_{\text {оп }}$ в соответствии с формулами (9)-(11).

Проведем аналогичный численный эксперимент и соответствующий расчет с исходными данными: $U_{\mathrm{Cmax}}=2 U_{\text {оп }}$, т. е. $N=2$. Вычисления осуществляем по соотношениям (9)-(11):

$$
\begin{gathered}
C_{\text {sum }}=\frac{t_{\text {imp }} \cdot j_{\text {max }}}{\left(U_{\text {on }}\right)}=2500 \mathrm{H} \Phi, \\
f_{\text {max }}=\frac{1}{T}=\frac{1}{t_{\text {imp }}} \cdot \frac{(N-1)}{(2 N-1)}=\frac{1}{3 \cdot 5 \text { мкс }}=66,7 \text { кГц. }
\end{gathered}
$$

Максимальная частота составит 66,55 кГц, а максимальная амплитуда заряда конденсатора составляет $2 \mathrm{~B}$, или два опорных напряжения $U_{\text {оп }}$.

Можно сделать вывод, что выражения для максимальной частоты и интегрирующего конденсатора, в зависимости от параметров системы, описываемой уравнениями (9)-(11), определены верно. 


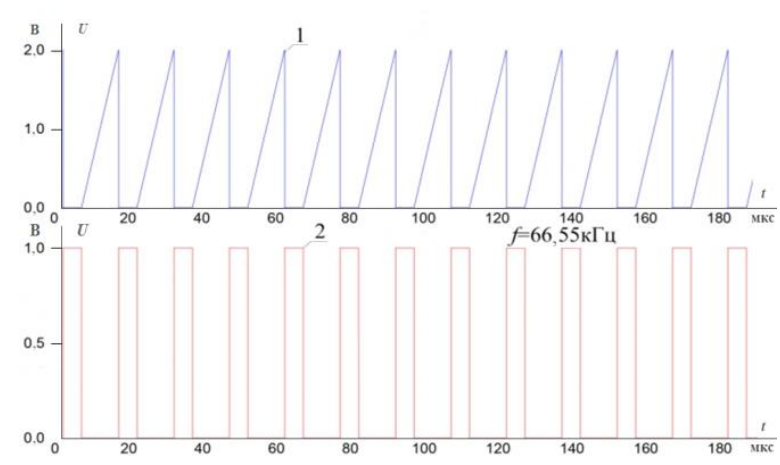

Pис. 8. Временные диаграммы при N=2: 1 - сигнал интегратора $\Theta(t) ; 2$ - импульсная последовательность $K F(\Theta)$

Fig. 8. Time diagrams for $N=2: 1$ - integrator signal $\Theta(t)$; 2 - pulse sequence $K F(\Theta)$

\section{Исследование динамики математической модели}

Для изучения установившихся режимов работы преобразователя с ЧИМ получены бифуркационные диаграммы посредством реализации стробоскопического отображения в Matlab [19], алгоритм которого приведен в [17]. Для сравнения стробоскопическое отображение построено также по результатам имитационного моделирования в LTSpice IV со следующей последовательностью действий $[16,17,20]$ :

- Проводится расчет выходного напряжения, полученные значения привязываются к временным точкам $t_{n}$ при формировании выходного массива данных.

- Из массива отсекается переходный режим. В оставшейся части выделяются точки напряжения $t_{n}$, находящиеся на месте спада коммутационной функции.

- Массив состояний в точках стробоскопического отображения сохраняется для некоторого, выбранного в качестве варьируемого, параметра, и строится бифуркационная диаграмма, представляющая из себя зависимость массива состояний $X$ системы от данного параметра.

На рис. 9 приводится бифуркационная диаграмма $[16,17,20]$, представляющая для $N=6$ качественные параметры установившегося режима в зависимости от коэффициента усиления $\alpha$ регулятора (рис. 5).

Итоги моделирования (рис. 9) показывают, что при значениях параметра $\alpha$ меньше величины 3,2 большинство точек соответствуют одночастотному режиму типа меандр, мгновенные значения вектора неизвестных $X$ и коммутационной функции $K F$ для которых приведены на рис. 10, $a$, т. е. в установившемся режиме работы стабилизатора управляющий сигнал не разбивается на пакеты импульсов. Далее с ростом $\alpha$ преобладает пакетный режим (рис. $10, \sigma)$.

По биффуркационной диаграмме четко видны аномальные явления в ПН с ЧИМ, при которых происходит качественное изменение установившегося режима - бифуркация деления периода, переход от номинального режима работы к аномальному, но детерминированному периодическому движению, при котором импульсы подаются пакетами (рис. 10, б).

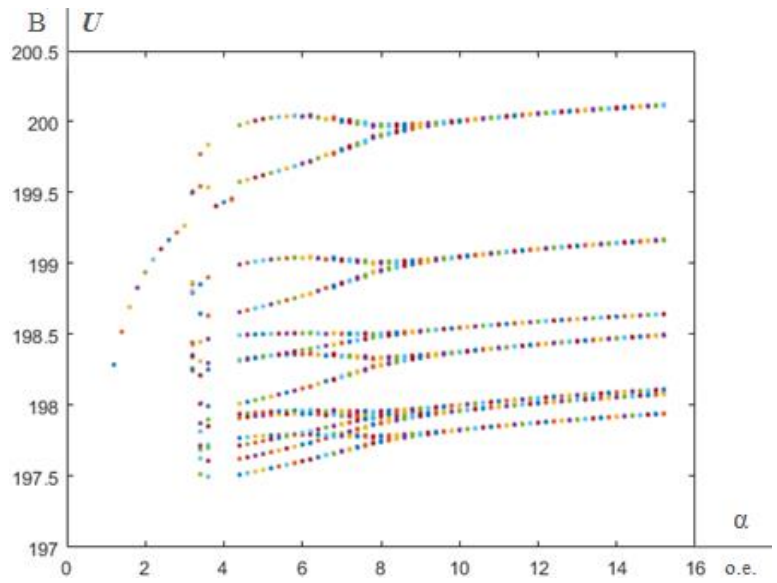

Pис. 9. Установивииеся режимы стабилизатора напряжения и их представление на бифуркачионной диаграмме (расчет при нулевых начальных условиях)

Fig. 9. Steady modes of the voltage stabilizer and their representation on the bifurcation diagram (zero initial conditions calculation)
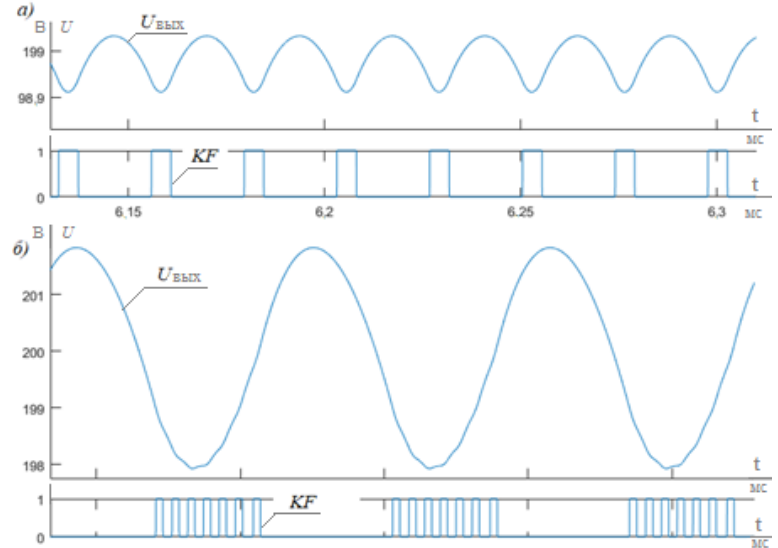

Pис. 10. Форма выходного сигнала и коммутационной функции: а) при $\alpha=2$, режим без разбиения на пакетьг; б) при $\alpha=15$, режим с разбиением на пакеть

Fig. 10. Form of the output signal and switching function at $\alpha=2$, non-packeted mode; $b)$ at $\alpha=15$, packeted mode

Построены также бифуркационные диаграммы для Csum=600 н $\Phi(N=5,2)$ (рис. 11) и Csum=2500 нФ $(N=2)$ (рис. 12) с локально изменяющимися начальными условиями.

Бифуркационные режимы (рис. 9-12) построены как посредством математической модели (2)-(4), (14)-(16), запрограммированной и вычисленной в среде Matlab, так и по имитационной модели в среде LTSpice IV. Результаты расчетов совпали как по качественным, так и по количественным значениям в математической и имитационной моделях с учетом принятых допущений [16-18] и погрешности численного метода системы автоматического проектирования. Количество линий на бифуркационной диаграмме в пакетном режиме равно числу импульсов в такте.

Бифуркационные диаграммы, приведенные на рис. 9-12, определяют области устойчивого функционирования преобразователя с точки зрения классической теории устойчивости Ляпунова - для номи- 
нального режима работы (представленного на рисунке одноцикловым сигналом) комплексные мультипликаторы Ляпунова располагаются внутри единичной окружности, т. е. критерий устойчивости выполняется. Таким образом, данного вида бифуркационные диаграммы могут рассматриваться как графическое представление зоны устойчивого функционирования стабилизатора напряжения ВГУ.

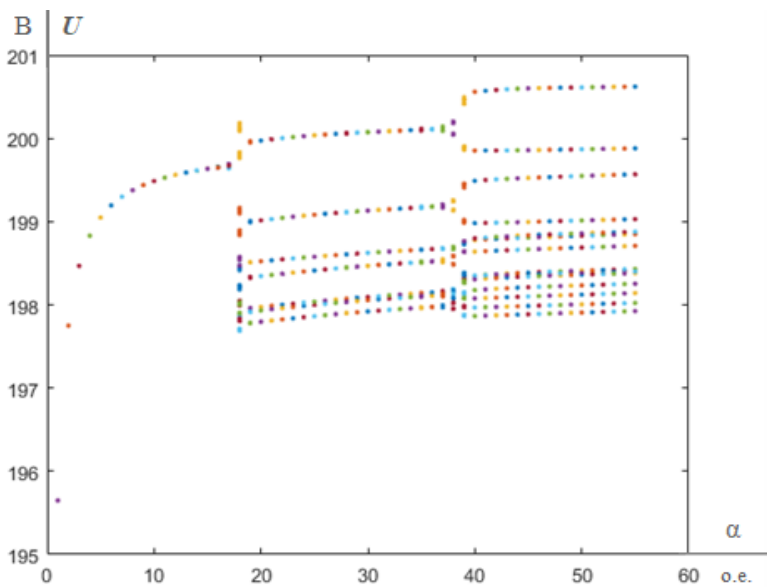

Рис. 11. Бифуркачионная диаграмма ПН с ЧИМ при $N=5,2$, расчет при локально изменяющихся начальных условиях

Fig. 11. Bifurcation diagram of voltage converter with a PFM at $N=5,2$, varying initial conditions calculation

Общими чертами нелинейной динамики импульсно-модуляционного преобразователя с ЧИМ по результатам исследований при изменении параметров системы (12) являются:

- В многомерном пространстве параметров всегда существует область нормального режима рабо$m b l$, в которой должна находиться рабочая точка.

- Эта область не всегда односвязная, и она ограничена аномальными режимами, границы которых точки бифуркации.

- Преобладающим типом бифуркационного перехода для ПН с ЧИМ является бифуркаичя деления периода, при которой появляется пакетный топо-

\section{СПИСОК ЛИТЕРАТУРЬ}

1. GWEC Global statistic // Официальный сайт Global Wind Energy Council - GWEC. URL: http://gwec.net/globalfigures/graphs/ (дата обращения: 14.08.2020).

2. Постановление Правительства РФ от 28 мая 2013 № 449 «О механизме стимулирования использования возобновляемых источников энергии на оптовом рынке электрической энергии и мощности» // ЭПС «Система ГАРАHT». URL: http://base.garant.ru/70388616/ (дата обращения: 14.08.2020).

3. Распоряжение Правительства РФ от 05 мая 2016 № 850-р «О продлении программы поддержки ВИЭ до 2024 года» // CПС КонсультантПлюс. URL: http://www.consultant.ru/ document/cons_doc_LAW_197712/ (дата обращения: 15.08.2020).

4. Брызгунов И.М. Без иллюзий. Вся правда о перспективах российской ветроиндустрии // Официальный сайт Российской ассоциации ветроиндустрии РАВИ. URL: https://rawi.ru/ru/bezillyuziy-vsya-pravda-o-perspektivah-rossiyskoy-vetroindustrii/ (дата обращения: 15.08.2020).

5. Жихарев А. Оценка эффектов реализации программы поддержки ВИЭ до 2024 г. // ООО «ВЫГОН Консалтинг». URL http://vygon.consulting (дата обращения: 16.08.2020). логический режим, определяемый пакетами импульсов коммутационной функции.

- Изменение характера управления на пакетный вариант приводит к росту амплитуды пульсаций выходного сигнала.

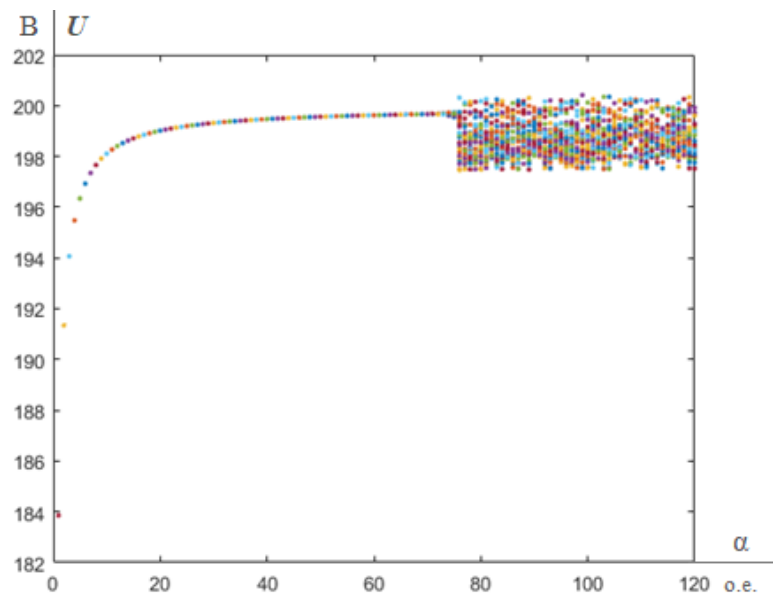

Puc. 12. Бифуркачионная диаграмма ПН с ЧИМ при $N=2$, расчет при локально изменяющихся начальных условиях

Fig. 12. Bifurcation diagram of voltage converter with a PFM at $N=2$, varying initial conditions calculation

\section{Заключение}

Рассмотрены режимы работы стабилизатора с изучением влияния его параметров на функционирование системы на базе понижающего преобразователя с ЧИМ. Рассмотрено влияние параметров системы на режимы работы. Получены бифуркационные диаграммы для стабилизатора и разных параметров структуры управления. Показано одновременное наличие номинальных и пакетных режимов, описана бифуркация деления периода, которая может представлять опасность для устойчивой работы ВГУ. Границы области номинального режима работы преобразователя в многопараметрическом пространстве, представленные в виде бифуркационных диаграмм, описывают зону его устойчивости функционирования.

6. Yaramasu V., Wu B., Sen P.C. High-power wind energy conversion systems: state-of-the-art and emerging technologies // Proceedings of the IEE - 2015 - V. 103 - Iss. 5 - P. 740-788.

7. ГОСТ Р 54418.1-2012 (МЭК 61400-1:2005) Возобновляемая энергетика. Ветроэнергетика. Установки ветроэнергетические. Ч. 1. Технические требования. - М.: Стандартинформ, 2016. $88 \mathrm{c}$.

8. Силовая электроника в энергопреобразующей аппаратуре альтернативной энергетики / Г.Я. Михальченко, С.Г. Михальченко, А.В. Миллер, В.В. Русанов. - Электропитание. - 2016. № 4. - C. 15-20.

9. Abir A., Mehdi D. Control of permanent-magnet generators applied to variable-speed wind-energy // 2017 International Conference on Green Energy Conversion Systems. - Hammamet, Tunisia, 2017. - P. 1-6

10. Stepins D. Effect of frequency modulation on input current of switch-mode power converter // IECON 2013 - 39th Annual Conference of the IEEE Industrial Electronics Society. - Vienna, Austria, 2013. - P. 683-688.

11. Русскин В.А., Семёнов С.М., Диксон Р.К. Исследование алгоритмов поиска точки максимальной мощности для повышаю- 
шего преобразователя напряжения солнечного инвертора Известия Томского политехнического университета. Инжиниринг георесурсов. - 2016. - Т. 327. - № 4. - С. 78-87.

12. Adaptive peak-inductor-current controlled PFM boost converter with a near-threshold startup voltage and high efficiency / H.H. Wu, C.L. Wei, Y.C. Hsu, R.B. Darling // IEEE Transactions on Power Electronics. - 2015. - V. 30. - Iss. 4. - P. 1956-1965.

13. Sudhakar C.J., Joshi D.R. Design of DC-DC converter for wind power application // 2019 Fifth International Conference on Electrical Energy Systems. - Chennai, India, 2019. - P. 1-4.

14. A buck converter operating in PFM mode, mathematical model and simulation analysis / A. Morra, M. Piselli, M. Flaibani, A. Gola // INTELEC 07 - 29th International Telecommunications Energy Conference. - Rome, Italy, 2007. - P. 23-26.

15. Side effects evaluation of switching frequency modulation in power converters / J. Mon, D. Gonzalez, J. Gago, J. Ballcells, A. Santolaria, L. Ferrer // IECON 2006 - 32nd Annual Conference on IEEE Industrial Electronics. - Paris, France, 2006. - P. 1643-1648.

16. Mikhalchenko G., Mikhalchenko S. Bifurcation behavior in multiparallel interleave buck converter // Control and Communications
(SIBCON), 2015 International Siberian Conference. - Omsk, Russia, 2015. - P. 1-7. DOI: 10.1109/SIBCON.2015.7147147.

17. Михальченко Г.Я., Михальченко С.Г. Математические модели импульсных систем преобразования энергии. - Томск: ТУСУР, 2013. $-138 \mathrm{c}$.

18. Tan R.H.G., Hoo L.Y.H. DC-DC converter modeling and simulation using state space approach // 2015 IEEE Conference on Energy Conversion. - Johor Bahru, Malaysia, 2015. - P. 42-47.

19. Shinde N., Sankad S., Patil S.L. Design and study voltage characteristics of buck converter by Matlab Simulink // 2018 2nd International Conference on Trends in Electronics and Informatics. - Tirunelveli, India, 2018. - P. 680-683.

20. Li S., Fahimi B., Kiani M. Bifurcation analysis and experimentation of peak current-controlled boost converter // IECON 2019 - 45th Annual Conference of the IEEE Industrial Electronics Society. - Lisbon, Portugal, 2019. - P. 1633-1638.

Поступила 04.12.2020 г.

\section{Информация об авторах}

Михальченко С.Г., доктор технических наук, заведующий кафедрой промышленной электроники Томского университета систем управления и радиоэлектроники.

Михальченко Г.Я., доктор технических наук, профессор кафедры промышленной электроники Томского университета систем управления и радиоэлектроники

Семенов С.M., старший преподаватель инженерной школы энергетики Национального исследовательского Томского политехнического университета.

Мещеряков П.С., доцент кафедры промышленной электроники Томского университета систем управления и радиоэлектроники.

Воронина Н.A., кандидат технических наук, доцент инженерной школы энергетики Национального исследовательского Томского политехнического университета.

Зюзев A.M., доктор технических наук, профессор кафедры электропривода и автоматизации промышленных установок Уральского федерального университета. 
UDC 621.314.1:621.382.2

\title{
BIFURCATION PHENOMENA IN VOLTAGE CONVERTER WITH FREQUENCY-PULSE CONTROL FOR A WIND GENERATOR
}

\author{
Sergey G. Mikhalchenko', \\ msg@ie.tusur.ru
}

Gennady Ya. Mikhalchenko

Sergey M. Semenov², ssm@tpu.ru

Pavel S. Meshcheryakov ${ }^{1}$, mps@ie.tusur.ru

\section{Nataliya A. Voronina ${ }^{2}$,} voronina@tpu.ru

Anatoliy M. Ziuzev ${ }^{3}$, a.m.zyuzev@urfu.ru

1 Tomsk State University of Control Systems and Radioelectronics, 40, Lenin avenue, Tomsk, 634050, Russia.

2 National Research Tomsk Polytechnic University, 30, Lenin avenue, Tomsk, 634050, Russia.

3 Ural Federal University,

19, Mira street, Ekaterinburg, 620002, Russia.

The relevance of the paper is determined by the need to improve energy efficiency parameters of power converting devices for the needs of alternative energy, in particular for a wind generator. One of the difficulties of converting wind energy is the low level of energy efficiency of this process. Due to the variable value of the rotational speed of the blades of the wind turbine in a certain time interval, the parameters of the received energy are variable, therefore, the settings of the secondary converters of electric energy must correspond to the time intervals for generating the signal. Improving the functioning of alternative power sources is possible through the use of multi-frequency stabilization modes of the converter. The article considers a model of a pulse-modulation step-down voltage converter based on pulse-frequency modulation. The authors have studied and proposed the methods for changing the width of the working zone of a singlefrequency stabilization mode.

The main aim of the study is to analyze the changing modes of the secondary voltage converter of a wind generator and to develop approaches to the study of bifurcation phenomena that occur in a converter with pulse-frequency modulation by constructing a mathematical model and numerically-analytical modeling of a buck voltage converter to improve the reliability of the system under consideration.

Methods: study of literature on standard energy conversion schemes in wind turbines with an intermediate DC link; mathematical modeling and conducting numerical experiments to change the operational parameters of a buck converter with pulse-frequency modulation to identify bifurcation in it; construction of bifurcation diagrams for various parameters of the transducer in order to increase the reliability of operation.

The results. The authors obtained the mathematical models and numerical-analytical technique for constructing and identifying dynamic modes of a voltage converter with pulse-frequency modulation acting as a DC link of a wind generator set and constructed the bifurcation diagrams for voltage stabilization at different parameters of the control system. The simultaneous presence of single-frequency and burstpacked modes was shown, bifurcation of period division was identified; the influence of the system parameters on the operating modes was studied, and methods for changing the width of the working zone of the single-frequency stabilization mode were proposed.

\section{Key words:}

Wind generation, buck voltage converter, pulse-frequency modulation, switching function, bifurcation diagram, single-frequency mode of stabilization.

\section{REFERENCES}

1. GWEC Global statistic. Official site of the Global Wind Energy Council - GWEC. Available at: http://gwec.net/globalfigures/graphs/ (accessed 14 September 2020).

2. Postanovlenie Pravitelstva RF ot 28 maya 2013 no. $449 \ll O$ mekhanizme stimulirovaniya ispolzovaniya vozobnovlyaemykh istochnikov energii na optovom rynke elektricheskoy energii $\mathrm{i}$ moshchnosti» [Decree of the Government of the Russian Federation of May 28, 2013 no. 449 «On the mechanism for stimulating the use of renewable energy sources in the wholesale electric and power»]. Electronic directory Garant. Available at: http://base.garant.ru/70388616/ (accessed 14 September 2020).
3. Postanovlenie Pravitelstva RF ot 05 maya 2016 no. $850-p \ll 0$ prodlenii programmy podderzhki VIE do 2024 goda» [Decree of the Government of the Russian Federation of May 05, 2016 №. 850-R «On extending the RES support program until 2024»]. Reference system KonsultantPlyus. Available at: http://www.consultant.ru/ document/cons_doc_LAW_197712/(accessed 15 September 2020).

4. Bryzgunov I.M. Bez illyuziy. Vsya Pravda o perspektivakh rossiyskoy vetroindustrii [No illusions. The whole truth about the prospects of the Russian wind industry]. Official website of the Russian Association of Wind Industry. Available at: https://rawi.ru/ru/bez-illyuziy-vsya-pravda-o-perspektivahrossiyskoy-vetroindustrii/ (accessed 15 September 2020). 
5. Zhikharev A. Otsenka effektov realizatsii programmy podderzhki VIE do 2024 [Evaluation of the effects of implementing the RES support program until 2024]. VYGON Consulting. Available at: http://vygon.consulting (accessed 16 September 2020)

6. Yaramasu V., Wu B., Sen P.C. High-power wind energy conversion systems: state-of-the-art and emerging technologies. Proceedings of the IEE, 2015, vol. 103, Iss. 5, pp. 740-788.

7. GOST R 54418.1-2012 (IEC 61400-1:2005) Vozobnovlyaemaya energetika. Vetroenergetika. Ustanovki vetroenergeticheskie. Chast 1. Tekhnicheskie trebovaniya. [State Standart 54418.1-2012. Renewable power engineering. Wind power engineering. Wind turbines. Part 1. Technical requirements]. Moscow, StandardInform Publ., 2016. 88 p.

8. Mikhalchenko G.Y., Mihalchenko, S.G., Miller, A.V., Rusanov V.V. Power electronics in energy-transforming equipment of alternative energy. Power Supply, 2016, Iss. 4, pp. 12-20. In Rus.

9. Abir A., Mehdi D. Control of permanent magnet generators applied to variable-speed wind-energy. 2017 International Conference on Green Energy Conversion Systems. Hammamet, Tunisia, 2017. pp. 1-6.

10. Stepins D. Effect of frequency modulation on input current of switch-mode power converter. IECON $2013-39^{\text {th }}$ Annual Conference of the IEEE Industrial Electronics Society. Vienna, Austria, 2013. pp. 683-688.

11. Russkin V.A., Semenov S.M., Dixon R.K. Construction of the adaptive algorithm of extreme regulation of power in the system of solar energy. Bulletin of the Tomsk Polytechnic University. Geo Assets Engineering, 2016, vol. 327, no. 4, pp. 78-87. In Rus.

12. Wu H.H., Wei C.L., Hsu Y.C., Darling R.B. Adaptive peakinductor-current controlled PFM boost converter with a nearthreshold startup voltage and high efficiency. IEEE Transactions on Power Electronics, 2015, vol. 30, Iss. 4, pp. 1956-1965.
13. Sudhakar C.J., Joshi D.R. Design of DC-DC converter for wind power application. 2019 Fifth International Conference on Electrical Energy Systems. Chennai, India, 2019. pp. 1-4.

14. Morra A., Piselli M., Flaibani M., Gola A. A buck converter operating in PFM mode, mathematical model and simulation analysis. INTELEC $07-29^{\text {th }}$ International Telecommunications Energy Conference. Rome, Italy, 2007. pp. 23-26.

15. Mon J., Gonzalez D., Gago J., Ballcells J., Santolaria A., Ferrer L. Side effects evaluation of switching frequency modulation in power converters. IECON $2006-32^{\text {nd }}$ Annual Conference on IEEE Industrial Electronics. Paris, France, 2006. pp. 1643-1648.

16. Mikhalchenko G., Mikhalchenko S. Bifurcation behavior in multiparallel interleave buck converter. Control and Communications (SIBCON), 2015 International Siberian Conference. Omsk, Russia, 2015. pp. 1-7. DOI: $10.1109 /$ SIBCON.2015.7147147.

17. Mikhalchenko G.Ya., Mikhalchenko S.G. Matematicheskie modeli impulsnykh sistem preobrazovaniya energii [Mathematical models of pulsed energy conversion systems]. Tomsk, TUSUR Publ., 2013. $138 \mathrm{p}$.

18. Tan R.H.G., Hoo L.Y.H. DC-DC converter modeling and simulation using state space approach. 2015 IEEE Conference on Energy Conversion. Johor Bahru, Malaysia, 2015. pp. 42-47.

19. Shinde N., Sankad S., Patil S.L. Design and study voltage characteristics of buck converter by Matlab Simulink. $20182^{\text {nd }}$ International Conference on Trends in Electronics and Informatics. Tirunelveli, India, 2018. pp. 680-683.

20. Li S., Fahimi B., Kiani M. Bifurcation analysis and experimentation of peak current-controlled boost converter. IECON 2019 $45^{\text {th }}$ Annual Conference of the IEEE Industrial Electronics Society. Lisbon, Portugal, 2019. pp. 1633-1638.

Received: 4 December 2020.

\section{Information about the authors}

Sergey G. Mikhalchenko, Dr. Sc., head of Industry Electronics Department, Tomsk State University of Control Systems and Radioelectronics.

Gennady Ya. Mikhalchenko, Dr. Sc., professor, Tomsk State University of Control Systems and Radioelectronics. Sergey M. Semenov, senior lector, National Research Tomsk Polytechnic University.

Pavel S. Meshcheryakov, associate professor, Tomsk State University of Control Systems and Radioelectronics.

Nataliya A. Voronina, Cand. Sc., associate professor, National Research Tomsk Polytechnic University.

Anatoliy M. Ziuzev, Dr. Sc., professor, Ural Federal University. 